\title{
Investigation of Truncated Waveguides
}

\author{
N.P. Lourie, D.T. Chuss, R.M. Henry, E.J. Wollack
}

\begin{abstract}
The design, fabrication, and performance of truncated circular and square waveguide cross-sections are presented. An emphasis is placed upon numerical and experimental validation of simple analytical formulae that describe the propagation properties of these structures. A test component, a 90-degree phase shifter, was fabricated and tested at 30 GHz. The concepts explored can be directly applied in the design, synthesis and optimization of components in the microwave to sub-millimeter wavebands.
\end{abstract}

Key Words - Circuit Synthesis and Modeling, Polarization, Waveguide Components, Eigenvalue Calculation

\section{INTRODUCTION}

Square and circular waveguide are commonly used in antenna feed networks for dual polarization systems. Not only do these simple geometries lend themselves to analytical calculation but also to conventional methods of fabrication and alignment. Their high degree of symmetry enables one to achieve homogenous propagation for both polarization modes. Truncation of the guide crosssection under this condition enables the realization of broadband components for antenna applications [1], mode conversion [2,3], and polarization discrimination [4].

At times, however, it is desirable to intentionally introduce phase shifts between the two degenerate modes in the structure to alter the polarization state in this propagation environment. Differential loading (i.e., intentionally breaking the symmetry between the two propagation waveguide modes) is typically employed to achieve the desired birefringence [1]. In metallic 
adiabatic waveguide structures this can be achieved through the introduction of perturbations in the guide cross-section, differentially loading the guide walls with dielectric, or subwavelength corrugations. Approaches to achieve this condition with discrete discontinuities include waveguide apertures/irises or posts separated by quarter-wave delays. Electromagnetically similar configurations with pockets, ridges, and septa can also be employed.

In going from single waveguide elements to freespace arrays of such structures, symmetry imposes a useful correspondence [5] that has led to the waveguide simulator technique [6] commonly used in the design of frequency- and polarization- selective surfaces. Examples that arise in practice include artificial dielectric layers [7,8], quasi-optical filters $[9,10]$, and multilayer absorber structures [11]. From perspective of a waveguide simulator, the frequency in units of guide cutoff frequency plays the role of the reciprocal of the sine of the scan angle in the modeled response. This mapping enables physical insight into the response of such waveguide and freespace analog structures. This is particularly useful in understanding the polarization response. For example, at a finite angle of incidence, one observes that the response of a dielectric (naturally-occurring or synthesized from guided-wave elements) is inherently birefringent. This can be viewed as an implicit constraint on the intrinsic polarization purity that can be achieved with uniform anti-reflection coating layers at finite incidence angle [8]. This interplay between the field's polarization state and the reflection balance of the two modes is a fundamental consequence of the underlying boundary conditions and is anticipated from the Fresnel coefficients at the interface and the symmetry of the artificial dielectric media [12]. As such, the techniques described have applicability beyond the simple example presented here.

In practice, it is useful to have accurate and simple analytical expressions to perform these initial steps in the design synthesis. Using a simple waveguide phase shifter as a worked example we present and validate analytical formulae for the cutoff wavelengths in truncated square and 
circular waveguides. The techniques introduced and demonstrated here at $30 \mathrm{GHz}$ can be extended to practical devices tailored to waveguide and quasi-optical applications through the THz spectrum.

\section{DESIGN APPROACH}

For dual-mode phase shifters it is desirable to achieve minimal and balanced reflection for both polarizations states. To realize this condition, the cutoff wavelength of the parent waveguide can be set equal to the average of those for each of the two dominant propagating modes, $\lambda_{c o} \approx\left(\lambda_{c}^{\prime \prime}+\lambda_{c}^{\perp}\right) / 2$. This can be seen by considering the magnitude of the discontinuity that results from each polarization's wave impedance relative to the parent guide.

When used as a quarter wave-plate, the input field's linear polarization is oriented 45-degrees with respect to the structure's principle-axis. The discontinuities are illuminated equally by both modes and the return loss is further symmetrized. Thus, by construction, the indicated condition can help in achieving a broadband match with minimal junction reactance and dispersion over the design band.

Stationary approximations can be employed to bound the magnitude of the dominant eigenvalue in the guide [13]. This approach is particularly useful in addressing problems for which the boundary or boundary conditions do not lend themselves to the usual method of separation of variables yet are closely related by a variation on a similar problem. In this spirit we estimate the eigenvalue for the dominant mode of propagation for the truncated structure,

$$
k_{c}^{2} \leq \frac{\int_{S}|\nabla \psi|^{2} d S}{\int_{S}|\psi|^{2} d S}
$$

where $\lambda_{c}=2 \pi / k_{c}$ is the cutoff wavelength in the guide and the trial function, $\psi$, satisfies the Helmholtz equation $[14,15]$ over the guide cross-section, $S$. The equality holds if the trial function 
is an eigenfunction for the structure. Thus, for configurations that are closely related by symmetry this approach provides a convenient means of deriving analytical expressions for use in design synthesis. ${ }^{1}$ For rectangular and circular guides we find this bound provides sufficient accuracy to specify an initial design geometry that achieves the desired propagation constants for practical structures.

The reflection imbalances commonly observed in truncated waveguide polarizer sections are not fundamental. A relatively simple variation on theme can be used to improve the performance. To illustrate this we consider a simple transmission line model of the structure by computing the parallel and perpendicular eigenvalues for truncated waveguides following [17,18]. For square guide with truncated corners we compute the cutoff wavelengths and find to lowest order:

$$
\begin{aligned}
& \frac{\lambda_{c}^{\perp}}{2 a} \approx \frac{1}{\sqrt{2}}\left(1+\frac{a^{\prime 2}}{2 a^{2}}\left[1-j_{o}^{2}\left(\pi a^{\prime} / a\right)\right]\right)+\ldots \\
& \frac{\lambda_{c}^{\prime \prime}}{2 a} \approx \frac{1}{\sqrt{2}}\left(1-\frac{a^{\prime 2}}{2 a^{2}}\left[1+j_{o}\left(\pi a^{\prime} / a\right)\right]^{2}\right)+\ldots
\end{aligned}
$$

where $a^{\prime} / a$ is the normalized guide truncation as depicted in Figure 1 and $j_{0}(x)=\sin (x) / x$ is the spherical Bessel function of order zero. The perpendicular case (2a) agrees with [15]; however, we find that the parallel case (2b) differs from the previously reported results.

Similarly, for circular guide with $N_{f}$ truncated facets we find:

$$
\frac{\lambda_{c}^{\perp}}{\lambda_{c}} \approx 1+\gamma_{\perp} N_{f} \cdot(\sin 2 \phi-2 \phi \cdot \cos 2 \phi)+\ldots
$$

\footnotetext{
${ }^{1}$ For example, the dimensions of circular and square cross-sections with equal cutoff frequency can be bounded by such considerations [16]. If the cylindrical section has radius $r$, the length of the side of a square section with equal area is $\pi^{1 / 2} r$. Equating areas under the assumption of similar modal symmetry one obtains an estimate of dominant circular cutoff wavelength, $\lambda_{c}\left(T E_{11}{ }^{o}\right) / 2 r$ $\approx \sqrt{ } \pi \approx 1.772$ which is within $\sim 4 \%$ of the actual value, $\lambda_{c}\left(T E_{11}{ }^{o}\right) / 2 r=\pi / \chi \approx 1.706$, where $\chi \approx 1.841$ is the first root of the $J_{1}{ }^{\prime}$. With similar accuracy the cutoff for a guide with hexagonal cross-section (i.e., $\theta=30^{\circ}, a^{\prime} / a=0.5$ ) may be estimated by comparison to a circular guide yielding, $\lambda_{c} / 2 a \approx(\pi / 2 \chi)(3 \sqrt{ } 3 / 2 \pi)^{1 / 2} \approx 0.776$. Such analytical bounds can be used to guide the assessment of the accuracy of numerical results.
} 


$$
\frac{\lambda_{c}^{\prime \prime}}{\lambda_{c}} \approx 1-\gamma_{\|} N_{f} \cdot(\sin 2 \phi-2 \phi \cdot \cos 2 \phi)+\ldots
$$

where $\lambda_{c}=\pi d / \chi \cong 1.706 d$ is the cutoff of a circular guide with diameter $d, \chi$ is the first root of $J_{1}{ }^{\prime}, \phi \equiv \cos ^{-1}(1-2 t / d)$ is specified by geometry depicted Figure 2, and the constants,

$$
\begin{aligned}
& \gamma_{\perp}=\frac{J_{1}^{2}(\chi) / 4 \pi \chi^{2}}{J_{1}^{2}(\chi)-J_{o}(\chi) \cdot J_{2}(\chi)} \approx 0.0333 \\
& \gamma_{\text {II }}=\frac{J_{1}^{2}(\chi) / 4 \pi}{J_{1}^{2}(\chi)-J_{o}(\chi) \cdot J_{2}(\chi)} \approx 0.1129
\end{aligned}
$$

are defined in terms of the Bessel functions, $J_{n}$, for notational convenience. We explore the accuracy of these analytical expressions by modeling representative guide structures with a TimeDomain Transmission-Line Matrix solver [19]. Comparisons of the analytical and simulated eigenvalues are presented in Figures 1 and 2 for truncated square and circular cross-sections. These results are in agreement with previous work [20,21].

The response of simple 90-degree phase shifter as a function of geometry provides an instructive example of the use and validations of these expressions. We use the computed eigenvalues to calculate the differential phase shift, $\Delta \theta=\left(k_{g}^{\prime \prime}-k_{g}^{\perp}\right) \cdot L$, for the parallel and perpendicular polarizations for a guide length, $L$. The guide $k$-vector for each mode is computed from $k_{g}=\left(2 \pi / \lambda_{o}\right) \cdot \sqrt{1-\left(\lambda_{o} / \lambda_{c}\right)^{2}}$ where $\lambda_{o}$ is the radiation wavelength in free space.

An estimate of the waveguide junction reactance is incorporated in the transmission line model to compute the influence of the interfaces between the homogenous guide and the phase 
shifter [22]. We find a single quarter-wave matching section at each end of the truncation is adequate to limit the influence of this discontinuity. The designs are summarized in Tables 1 and 2. The "Inhomogeneous" structures in the table maintain the cutoff of the parent guide geometry in one plane while for the " $<$ Homogenous $>$ " structures the propagation properties are balanced on average as previously described. This choice maximizes the birefringence for a targeted level of reflection and component's envelop.

\section{MEASUREMENTS}

A simple 90-degree phase shifter in circular guide based on the described design approach was fabricated in electroformed copper and evaluated to validate the eigenvalue estimates. The design parameters for the test structure are listed in the last line in Table 2, and a photograph of the prototype and calibration set is shown in Figure 3. Angular alignment of the electric field polarization in 45-degree increments was achieved with 0.0625 " diameter ground dowel pins and thru holes in the interface flanges. Centering of the circular waveguide interfaces was realized by clamping the outer diameter flange surfaces, which by construction, are centered on the guide. This flange configuration enabled measurement at selected orientations.

The response was measured with a Hewlett-Packard 8510 Vector Network Analyzer using a TRL (Thru-Reflect-Line) calibration. The thru standard's guide diameter and the test set interface transitions have the same cutoff as WR22.4. Measurements of the structure's reflectance and differential phase shift are presented in Figure 4 along with full-wave simulations of the structure's nominal design parameters.

The deviation between the observed response and the simulation predictions are consistent with the $\sim 25$ micron fabrication tolerances achieved in the realization of the electroformed waveguide. A summary of the design cutoff parameters for the structure is provided in Table 3. Spurious 
modal excitation was not noted during testing of the prototype structure, and the observed ohmic loss was within a factor of $\sim 1.16$ of that computed for a copper structure of the same dimensions

The accuracy of the transmission line circuit model is adequate but limited by the simplistic treatment of the junction's reactance. For a multi-section phase shifter the circuit simulation would be useful for initial design estimates, however, additional refinement would be required to achieve an unbiased phase shift estimate.

\section{CONCLUSION}

We present and validate analytical expressions that enable efficient computation of the propagation properties in truncated circular and square waveguides cross-sections. These equations allow efficient synthesis of dual-polarization components with balanced residual reflections. A simple phase shifter has been modeled, fabricated, and measured to validate the modeling techniques presented.

\section{ACKNOWLEDGMENT}

Mechanical design support provided by M. Limon is graciously acknowledged. 


\section{REFERENCES}

[1] J. Uher, J. Bornemann, U. Rosenberg, Waveguide Components for Antenna Feed Systems: Theory and CAD, Artech House, Norwood, MA, 419-435, 1993.

[2] J.R. Pyle, “A Circular-to-Rectangular Waveguide Transition Maintaining a Constant Cutoff Wavelength," Australian Defense Scientific Service Weapons Research Establishment Salisbury, S.A. Tech. Note PAD 94 (1964); Also see: J.R. Pyle and R.J. Ashley, "Cutoff Wavelengths of Waveguides with Unusual Cross Sections," IEEE Transactions on Microwave Theory and Techniques, Vol. MTT-12, (1964) 556-557; Correction to parallel case see: D.H. Sinnott, et al., "The Finite Difference Solution of Microwave Circuit Problems," IEEE Transactions on Microwave Theory and Techniques, Vol. MTT-17, No. 8 (1969), 464-478.

[3] D.A. Bathker, “A Stepped Mode Transducer Using Homogeneous Waveguides," IEEE Transactions on Microwave Theory and Techniques, Vol. MTT-15, No. 2 (1967), 128-130.

[4] E. Wollack and W. Grammer, "Symmetric Waveguide Orthomode Junctions," Proceedings of the 14th International Symposium on Space TeraHertz Technology, Tucson AZ, editors E. Walker and J. Payne (2003), 169-176.

[5] J.C. Slater, "Microwave Transmission," McGraw-Hill, NY, Chapter 7, 1942.

[6] R.C. Hansen, “Phased Array Antennas,” John Wiley \& Sons, Inc., New York, 458-464, 1998.

[7] R.E. Collins, "Field Theory of Guided Waves", second edition, IEEE Press, Piscataway, NJ, Chapter 12, 1990.

[8] P.-S. Kildal, et al., "Meniscus-Lens-Corrected Corrugated Horn: A Compact Feed for a Cassegrain Antenna”, IEE Proceedings, Vol. 131, Part H., No. 6 (1984), 390-394.

[9] J.W. Archer, "A Novel Quasi-Optical Frequency Multiplier Design for Millimeter and Submillimeter Wavelengths," IEEE Transactions on Microwave Theory and Techniques, Vol. 32, No. 4 (1984), 421-427. 
[10] P.A.R. Ade, G. Pisano, C. Tucker, S. Weaver, "A Review of Metal Mesh Filters", Millimeter and Submillimeter Detectors and Instrumentation for Astronomy III, edited by Jonas Zmuidzinas, Wayne S. Holland, Stafford Withington, William D. Duncan, Proc. of SPIE Vol. 6275 (2006), 62750U; doi: 10.1117/12.673162

[11] Wollack, E.J., Chuss, D.T., Moseley, S.H., "Electromagnetic Considerations for Planar Bolometer Arrays in the Single Mode Limit," Proceedings of the Society of Photo-Optical Instrumentation Engineers (SPIE), Millimeter and Submillimeter Detectors and Instrumentation for Astronomy, Editors J. Zmuidzinas, W.S. Holland and S. Withington, W.D. Duncan, Vol. III (2006), V2750.

[12] A. MacKay, "Proof of Polarization Independence and Nonexistence of Crosspolar Terms for Targets Presenting n-Fold ( $\mathrm{n}>2$ ) Rotational Symmetry with Special Reference to Frequency-Selective Surfaces,” Electron. Lett., Vol. 25, No. 24 (1989), 1624-1625.

[13] K.A. Milton and J. Schwinger, "Electromagnetic Radiation: Variational Methods, Waveguides and Accelerators," Springer-Verlag, Berlin, Chapter 10, 2006.

[14] C.G. Montgomery, R.H. Dicke, E.M. Purcell, Principles of Microwave Circuits, IEE Electromagnetic Waves Series, Vol. 25, Peter Peregrinus, London, Chapter 12, (First published 1948, MIT Radiation Laboratory Series, Vol. 8, McGraw-Hill, New York), section $2.15,42-45,1987$.

[15] W.R. Smythe, Static and Dynamic Electricity, Third Edition, 1968, McGraw-Hill, New York, 523-526, 1968.

[16] P.M. Morse and H. Feshbach, Methods of Theoretical Physics, 1953, McGraw-Hill, New York, 1131-1133, 1953.

[17] R. Levy, "The Relationship Between Dual Mode Cavity Cross-Coupling and Waveguide Polarizers," IEEE Transactions Microwave Theory and Techniques, Vol. MTT-43, No. 11 (1995), 2614-2620; Also see, R. Levy, “Correction to 'The Relationship Between Dual Mode 
Cavity Cross-Coupling and Waveguide Polarizers," IEEE Transactions Microwave Theory and Techniques, Vol. MTT-45, No. 5 (1997), 704-705.

[18] E.J. Wollack and F.M. Vanin, "Broadband Transitions for Micro-machined Waveguide," Proc. IEEE, MTT-S (2007), 1749 - 1752.

[19] CST (Computer Simulation Technology) MICROSTRIPES ${ }^{\mathrm{TM}}$, Framingham, MA 01701, United States.

[20] C. Y. Wang, "Frequencies of a Truncated Circular Waveguide-Method of Internal Matching", IEEE Trans. Microwave Theory Tech., Vol. 48, No. 10 (2000), 1763-1765.

[21] S.-L.-Lin, L-W. Li, T.-S. Yeo, M.-S., Leong, "Cutoff Wavenumbers in Truncated Waveguides", IEEE Microwave and Wireless Components Letters, Vol. 11, No. 5 (2001), 214-216.

[22] G. Matthaei, L. Young, E.M.T. Jones, Microwave Filters, Impedance-Matching Networks and Coupling Structures, McGraw-Hill, New York, 300-304, 1964. 
Figures

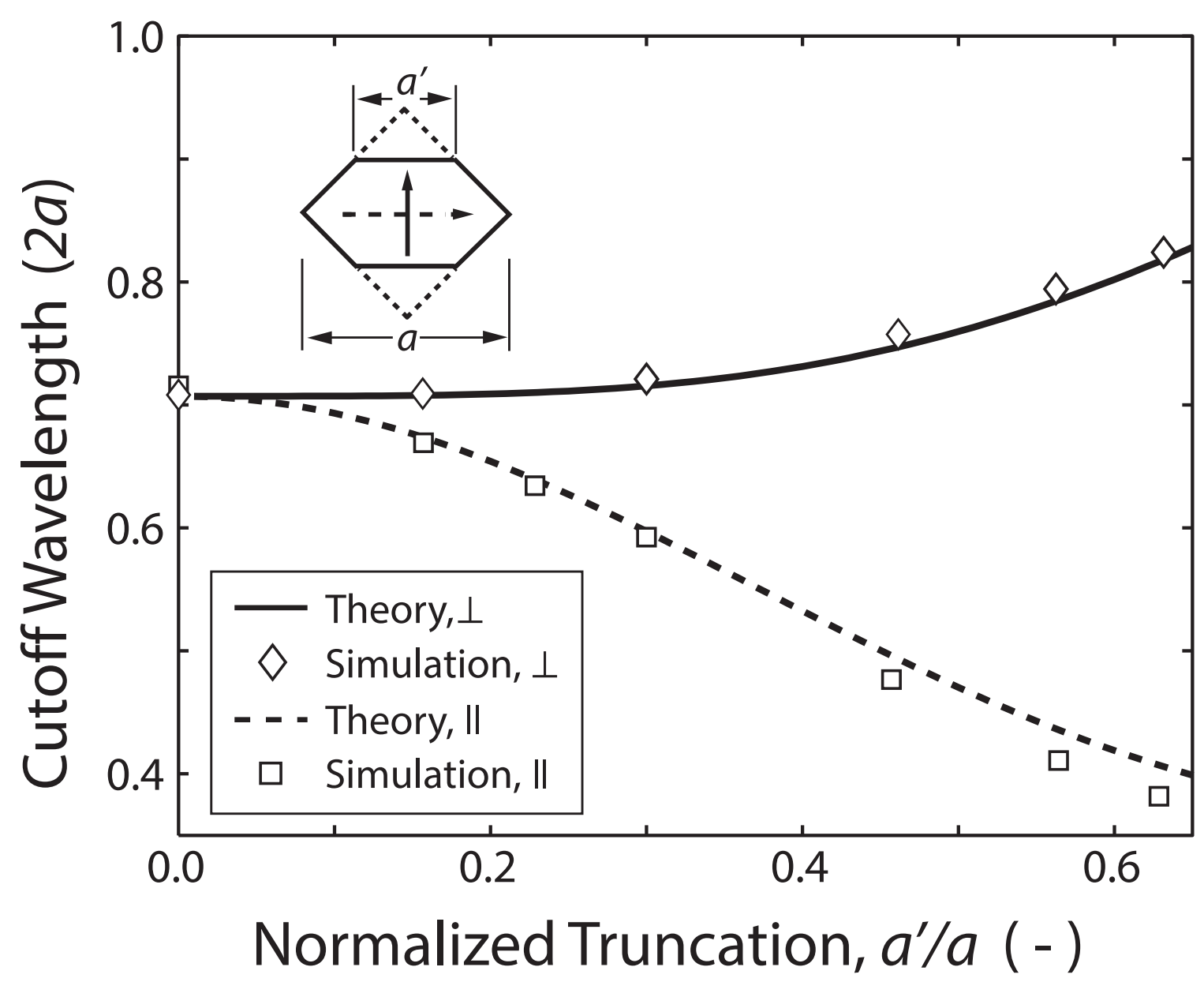

Fig. 1. Cutoff Wavelength for Symmetrically Truncated Square Guide. Data presented are from the perturbation theory calculations (solid and dashed lines) and Time-Domain TransmissionLine Matrix solver simulations (diamond and square symbols) for representative geometries. Figure inset depicts the truncated square guide geometry and orientation of electric field polarization vector. 


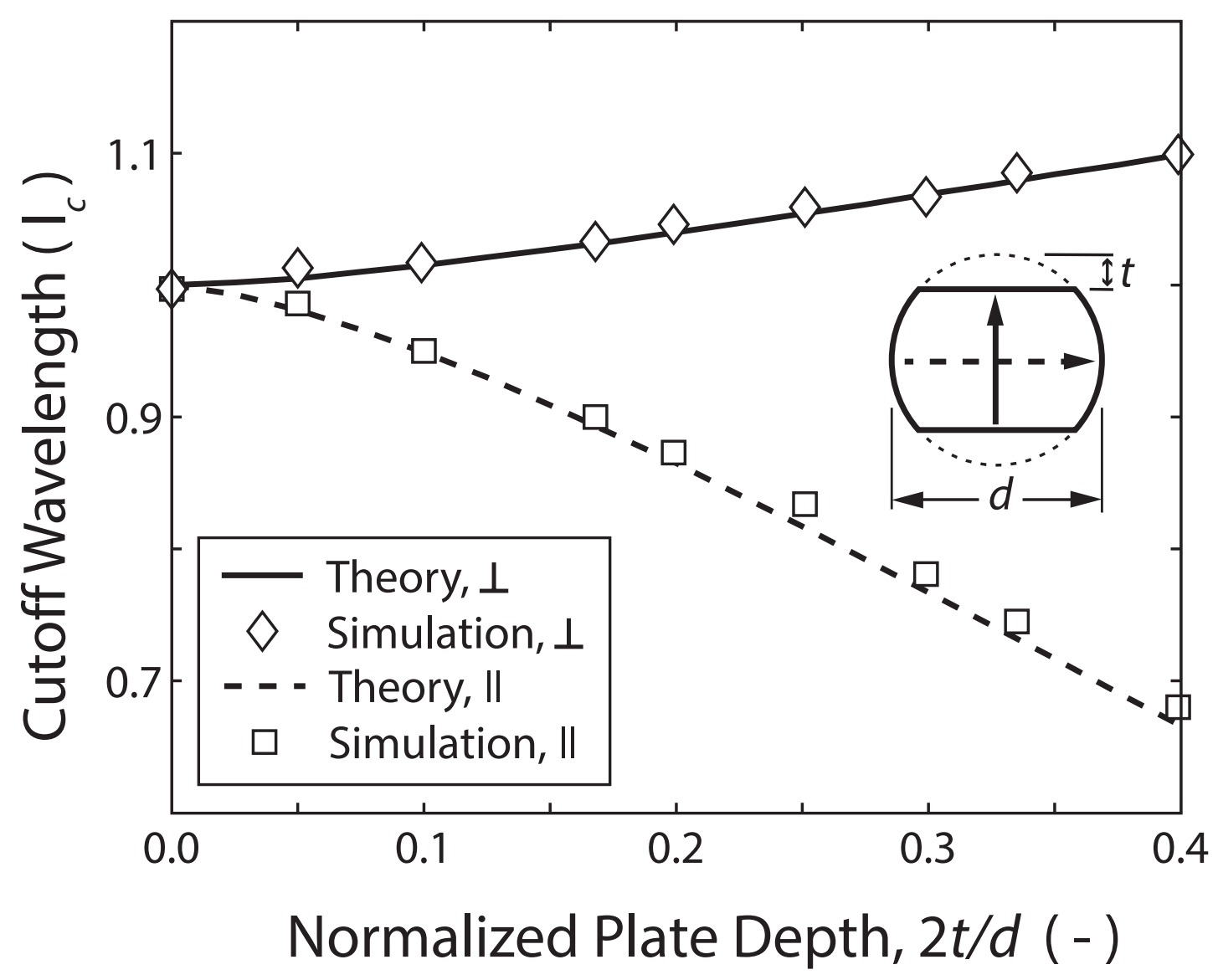

Fig. 2. Cutoff Wavelength for Symmetrically Truncated Circular Guide. Data presented are from perturbation theory calculations (solid and dashed lines) and Time-Domain Transmission-Line Matrix solver simulations (diamond and square symbols) for representative geometries. Figure inset depicts the truncated cicular guide geometry and orientation of electric field polarization vector. 


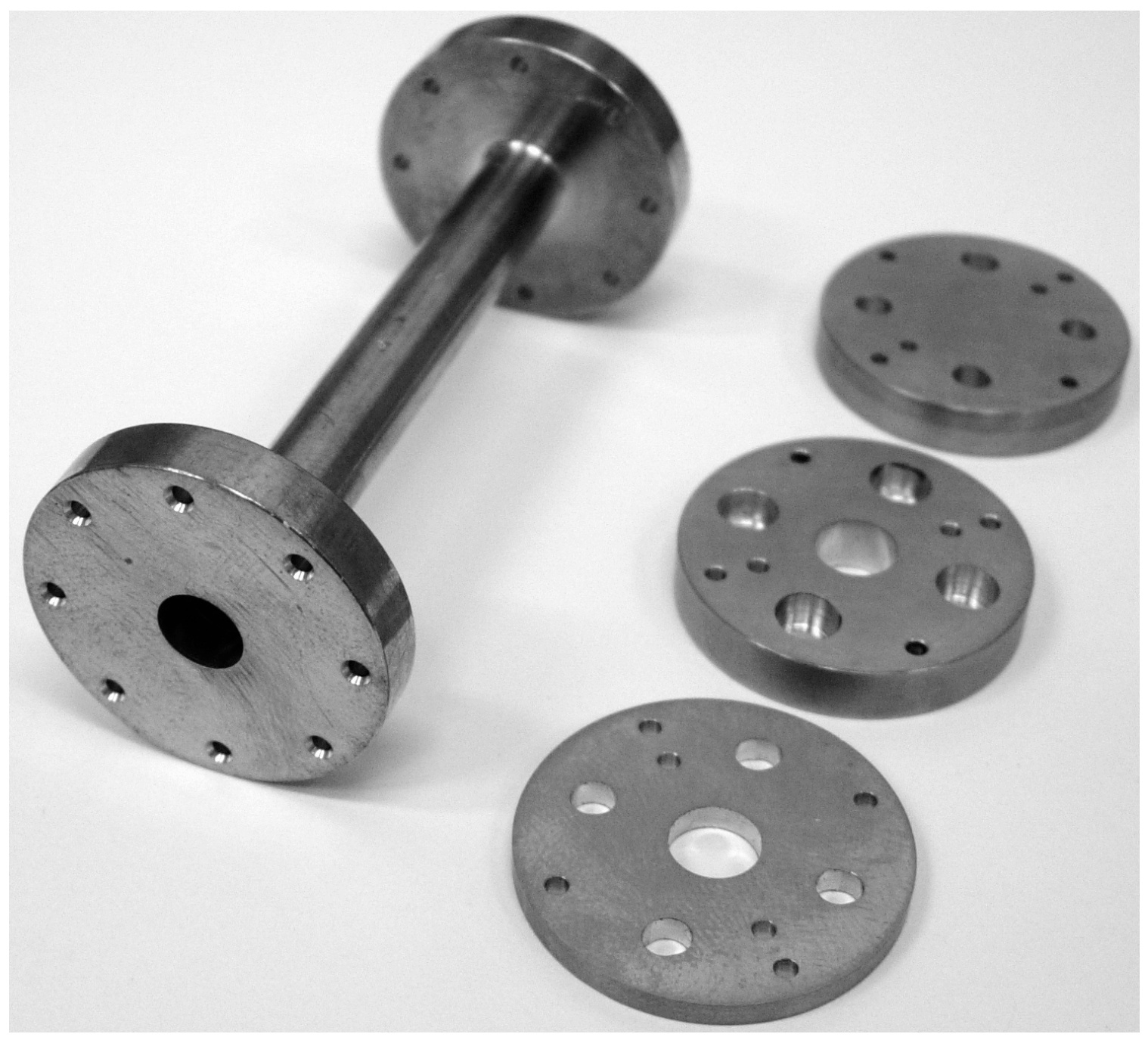

Fig. 3. Prototype Phase Shifter and TRL Calibration Standards. 

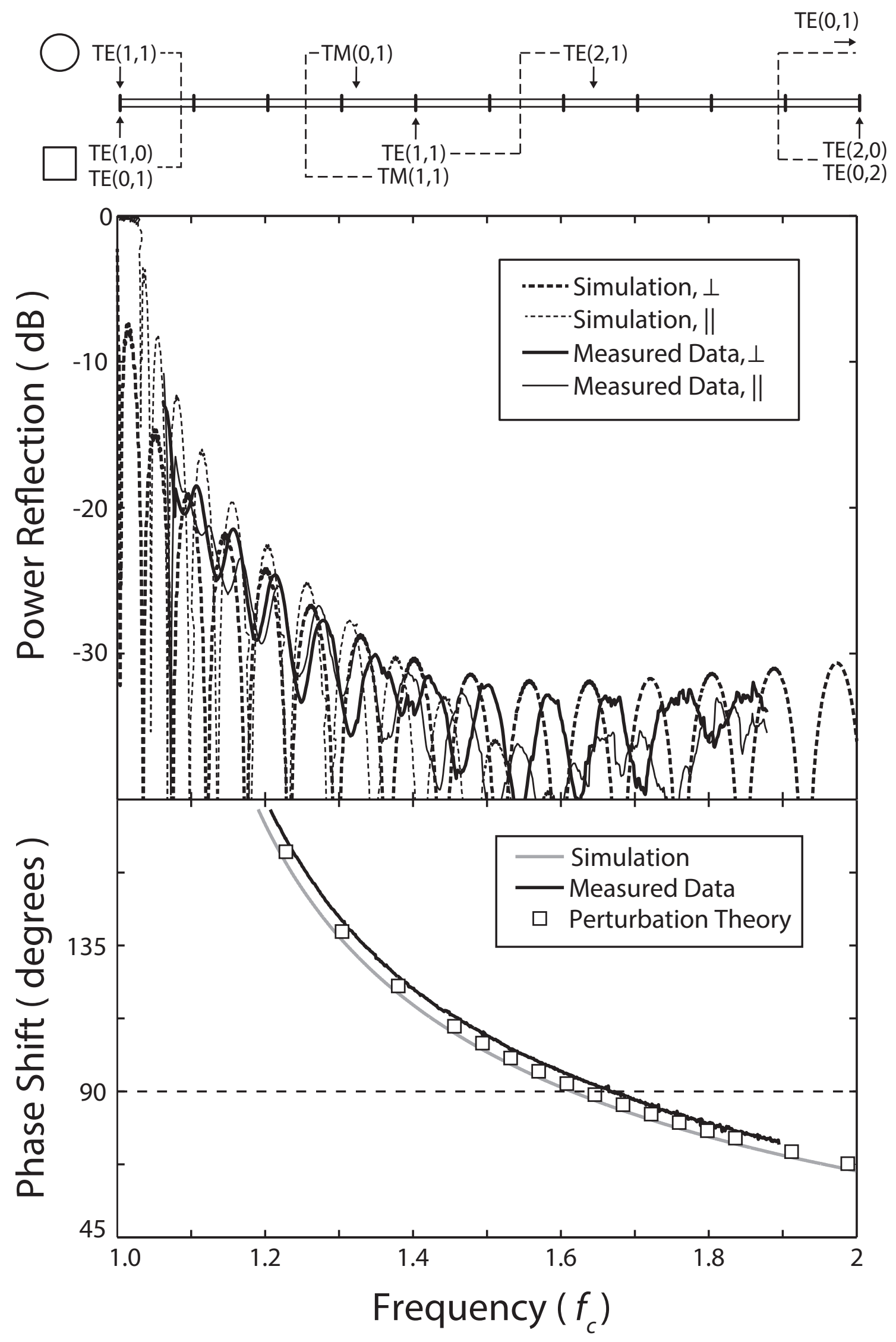
Fig. 4. Phase Shifter Reflection for Parallel and Perpendicular Illumination (Upper). Data from the numerical simulation (dashed) and measurement of structure (solid) for each polarization are plotted in the upper figure. The diagram across the top indicates the mapping between modes in circular and square guides having similar symmetry. From reviewing the mode map at the top of the figure, one notes that no spurious higher order modes are excited and trapped in the structure. Differential Phase Shift (Lower). Data are plotted for the perturbation theory parameters (square symbols), Time-Domain Transmission-Line Matrix solver simulations (grey line), and measurements of prototype structure (black line) in the lower figure. The cutoff frequency of parent guide and phase shifter length are $f_{c}=26.36 \mathrm{GHz}$ and $L=10 a$.

TABlE 1: TRUnCATED SQUARE QUARTER-WAVE

PHASE SHIFTER DESIGN SUMMARY

\begin{tabular}{ccccc}
\hline \hline Delay & Parent & Truncated & Reflection & \\
Length & Diagonal & Width & $@ \Delta \theta=\pi / 2$ & Notes: \\
$L / \lambda_{c}$ & $a / \lambda_{c}$ & $a^{\prime} / a$ & {$[\mathrm{~dB}]$} & \\
4.688 & 0.707 & 0.178 & -26 & Inhomogeneous \\
1.897 & 0.756 & 0.280 & -23 & $<$ Homogeneous $>$ \\
3.016 & 0.742 & 0.230 & -27 & $<$ Homogeneous $>$ \\
5.067 & 0.729 & 0.178 & -32 & $<$ Homogeneous $>$ \\
\hline \hline
\end{tabular}




\begin{tabular}{ccccc}
\hline \hline \multicolumn{5}{c}{ TABLE 2: TRUNCATED CIRCULAR QUARTER-WAVE } \\
& \multicolumn{4}{c}{ PHASE SHIFTER DESIGN SUMMARY } \\
\hline \hline Delay & Parent & Truncated & Reflection & Notes: \\
Length & Diameter & Step & @ $\Delta \theta=\pi / 2$ & \\
$L / \lambda_{c}$ & $d / \lambda_{c}$ & $t / d$ & {$[\mathrm{~dB}]$} & \\
4.737 & 0.586 & 0.049 & -24 & Inhomogeneous \\
2.002 & 0.611 & 0.090 & -23 & $<$ Homogeneous $>$ \\
2.920 & 0.604 & 0.070 & -27 & $<$ Homogeneous $>$ \\
4.955 & 0.597 & 0.049 & -31 & $<$ Homogeneous $>$ \\
\hline \hline
\end{tabular}

Table 3: Truncated Circular Phase Shifter

CUTOFF SUMmaRY

\begin{tabular}{|c|c|c|c|}
\hline & $\lambda_{c}[\mathrm{~mm}]$ & $\lambda_{c}\left[2 \mathrm{a}_{\mathrm{o}}\right]$ & $f_{c}[\mathrm{GHz}]$ \\
\hline E-Perpendicular Truncation & $11.76 \mathrm{~mm}$ & 1.033 & 25.52 \\
\hline E-Parrallel Truncation & $11.01 \mathrm{~mm}$ & 0.9679 & 27.24 \\
\hline Average Perpendicular / Parallel & $11.38 \mathrm{~mm}$ & 1.001 & 26.35 \\
\hline Parent Homogenous Guide & $11.38 \mathrm{~mm}$ & 1.000 & 26.36 \\
\hline
\end{tabular}

